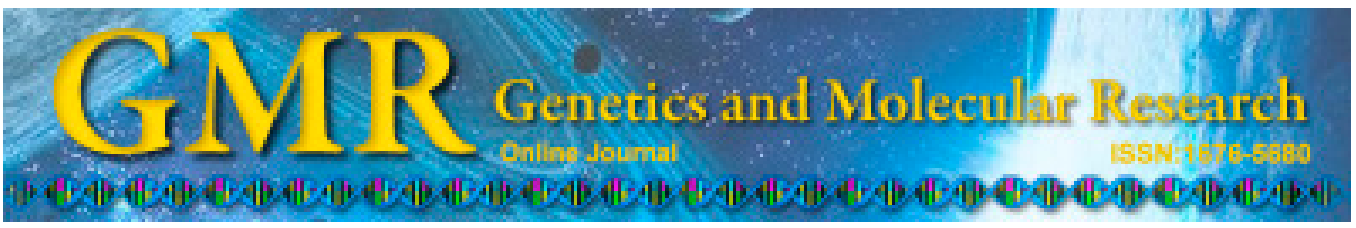

\title{
Cut-and-paste-based cloning strategy for large gene site-directed mutagenesis
}

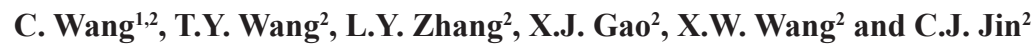 \\ ${ }^{1}$ Department of Hematology, \\ the First Affiliated Hospital of Zhengzhou University, Zhengzhou, China \\ ${ }^{2}$ Anhui Key Laboratory for Cellular Dynamics and Chemical Biology, \\ Hefei National Laboratory for Physical Sciences at the Microscale and School \\ of Life Sciences, University of Science and Technology of China, Hefei, China \\ Corresponding author: C.J. Jin \\ E-mail: yanfangliucn@163.com
}

Genet. Mol. Res. 14 (2): 5585-5591 (2015)

Received July 29, 2014

Accepted January 19, 2015

Published May 25, 2015

DOI http://dx.doi.org/10.4238/2015.May.25.10

\begin{abstract}
Site-directed mutagenesis is an essential technique for investigating the mechanisms of gene regulation on a molecular level, as well as for exploring post-translational modifications and functional structure at the protein level. Polymerase chain reaction combining in vitro synthesis of oligonucleotide primers allows for site-directed mutation to be performed with ease. However, site-directed mutagenesis is difficult when larger plasmids are involved. Here, we present a novel method for generating large gene site-directed mutagenesis products based on a cut-and-paste-based cloning strategy. This method uses 4 primers, incorporating relevant mutations and restriction enzyme site sequences, to generate 2 DNA fragments by polymerase chain reaction. The fragments are then ligated into TA cloning vectors. Large genes containing mutations of interest were obtained by cutting and then pasting, and then inserting one fragment into another T-vector. We demonstrated the practicality of this method by creating a G59S mutation within the $\mathrm{p} 150^{\text {Glued-encoding gene. }}$
\end{abstract}

Key words: Cut-and-paste-based cloning strategy; $\mathrm{p} 150^{\text {Glued; }}$ Polymerase chain reaction; Site-directed mutagenesis 


\section{INTRODUCTION}

Site-directed mutagenesis, as well as nucleotide insertions, deletions, and substitutions, have been extensively used in studies examining the essential molecular mechanisms of gene and protein function. Two revolutionary techniques, in vitro synthesis of oligonucleotides and polymerase chain reaction (PCR), make site-directed mutagenesis a robust and versatile method. In vitro synthesis of oligonucleotides ensures that novel point mutations are available in a prearranged manner at a specific site (Hutchison 3rd et al., 1978). Moreover, the simultaneous use of PCR facilitates increased versatility and efficiency of site-directed mutagenesis (Zoller and Smith, 1982). Thus, over the past few decades, PCR-driven DNA mutagenesis has improved in its simplicity, efficiency, and versatility (Higuchi et al., 1988; Ho et al., 1989; Hemsley et al., 1989; Sang et al., 1996; Nabavi and Nazar, 2005; Jin et al., 2007; Heckman and Pease, 2007; Carrigan et al., 2011; Bok and Keller, 2012; Munteanu et al., 2012; Liang et al., 2012; Wei et al., 2012; Yan et al., 2012; Bu et al., 2013; Mitchell et al., 2013).

PCR-mediated overlap extension site-directed mutagenesis was developed independently by 2 research groups in the late 1980s (Higuchi et al., 1988; Ho et al., 1989). This method employs 2 initial PCRs to create 2 overlapping segments using 2 internal mutagenic overlapprimers. During the second PCR, heteroduplexes extend via overlapping sequences to generate a full-length double-stranded mutant DNA. However, the main limitations of this method include its ineffectiveness for use in genes larger than $2 \mathrm{~kb}$ or when the intended mutations are near gene ends (Higuchi et al., 1988; Ho et al., 1989; Heckman and Pease, 2007). Megaprimer mutagenesis employs a similar strategy as the overlap-extension method, except that it requires only 1 internal mutagenic primer (Nabavi and Nazar, 2005). Both PCR-mediated overlap extension and megaprimer mutagenesis require subcloning of a mutant gene into a vector.

Inverse PCR site-directed mutagenesis is a straightforward technique used to linearly amplify the entire double-stranded plasmid with a pair of back-to-back primers, and then circularize the primer by ligating the 2 blunt-ends (Hemsley et al., 1989; Sang et al., 1996). This is a simple and rapid method for inserting mutations anywhere in a plasmid, but is limited for use in smaller plasmids. Furthermore, amplification of the entire plasmid requires a quality DNA polymerase with higher efficiency and fidelity.

In this study, we developed a novel method for generating large gene site-directed mutagenesis, which we have termed cut-and-paste-based cloning strategy (CPCS). Briefly, fulllength DNA was split into 2 fragments containing the desired mutations, amplified by PCR, and then subcloned into T-vectors. The full-length mutant was obtained by cutting 1 fragment from 1 vector and pasting it into another fragment-containing vector. We validated the practicality of this method by introducing a G59S mutation within the $\mathrm{p} 150^{\text {Glued-encoding gene. }}$

\section{MATERIAL AND METHODS}

\section{Primers}

Sequence P150a 5'-GAATTCAATGGCCCAGAGCAAGAGG-3', P150c 5'-CTCGA GGATCACGCTCACCCACTTGCCAGTGGC-3', P150b 5'-GAATTCGTCGACGAAGCAA AAGGCAAGAAC-3', and P150d 5'-CTCGAGTTAGGAGATGAGACGACCGTG-3' were synthesized as primers (restriction sites are in boldface; same-sense mutation sites are in boldface and enclosed in a border; desired mutation site is in boldface and italic). 


\section{Cell culture and transfection}

HeLa cells from American Type Culture Collection (Manassas, VA, USA) were cultured as subconfluent monolayers in Dulbecco's modified Eagle's medium (Invitrogen, Carlsbad, CA, USA) containing 10\% fetal bovine serum (Hyclone, Logan, UT, USA), $100 \mathrm{U} / \mathrm{mL}$ penicillin, and $100 \mu \mathrm{g} / \mathrm{mL}$ streptomycin (Invitrogen) under $10 \% \mathrm{CO}_{2}$. Flag-p150 ${ }^{\text {Glued }}$ wild-type (WT) and G59S plasmids were transfected into HeLa cells using Lipofectamine 2000 (Invitrogen) reagent according to manufacturer instructions.

\section{Western blotting}

HeLa cells expressing Flag-p150 Glued WT and G59S mutant were fixed in sample buffer, subjected to $10 \%$ sodium dodecyl sulfate-polyacrylamide gel electrophoresis, transferred to a nitrocellulose membrane, probed with anti-FLAG (M2) antibody (Sigma-Aldrich, St. Louis, MO, USA), and developed using an electrochemiluminescence detection kit.

\section{RESULTS}

\section{Large gene mutagenesis method based on CPCS}

An overview of our mutagenesis procedure is presented as a schematic diagram in Figure 1. First, 2 target gene segments were amplified from template DNA by PCR using 4 primers, including 2 flanking primers (a and d). Next, restriction enzyme sites (R1 and R4) were incorporated; the 5'-ends of both DNA strands were marked and used as the 2 internal primers ( $b$ and $c$ ). The mutation of interest (indicated by a star) and/or a same-sense mutation were then introduced to create restriction enzyme sites (R2 and R3, which may use the same restriction enzyme site or have homologous ends or blunt ends). Next, 2 PCR fragments were TA-subcloned into T-vectors. Finally, 1 fragment was cut (R1 and R2) and inserted into the T-vector (predigested by R1 and R3) containing another fragment. Thus, a large gene with the desired mutation was obtained in a T-vector. This method can be applied to generate large cDNAs of fusion proteins, chimeric proteins, or deletions. Precise design of primers is crucial for the success of this method. Specific software programs, such as SiteFind and Primer Generator (Turchin and Lawler Jr., 1999; Evans and Liu, 2005), can be used for the design of a restriction enzyme site within the mutation primers without altering the translated amino acid sequence.

\section{Application of this method to the $150^{\text {Glued }}$ G59S mutation}

p150 Glued is a major component of dynein/dynactin complexes and binds to dynein and microtubules (Puls et al., 2003). The G59S mutation within the conserved microtubule-binding domain of $\mathrm{p} 150^{\text {Glued }}$ leads to aberrant aggregation of $\mathrm{p} 150^{\text {Glued }}$, impaired dynactin function, defected vesicular trafficking, induction of neuronal cell death, and an autosomal dominant form of motor neuron disease (Puls et al., 2003; Levy et al., 2006; Laird et al., 2008). Recently, we found that F-box/LRR-repeat protein 5 binds to and promotes poly-ubiquitination and protein turnover of $\mathrm{p} 150^{\text {Glued }}$ (Zhang et al., 2007). We propose that F-box/LRR-repeat protein 5 is unable to interact with G59S p150 Glued and therefore cannot regulate its subsequent degradation, resulting in aggregation of G59S p150 Glued. We generated a $150^{\text {Glued }}$ G59S mutation 
to examine this hypothesis.

The full-length coding sequence of rat $\mathrm{p} 150^{\text {Glued }}$ (GenBank accession No. X62160) is $3843 \mathrm{bp}$ in length. Although other methods of site-directed mutagenesis have been applied to large genes with some success (Munteanu et al., 2012), this process remains difficult. Moreover, as DNA length increases, the risk of incorporating additional undesired mutations also increases. Thus, we developed a CPCS for large gene site-directed mutagenesis.

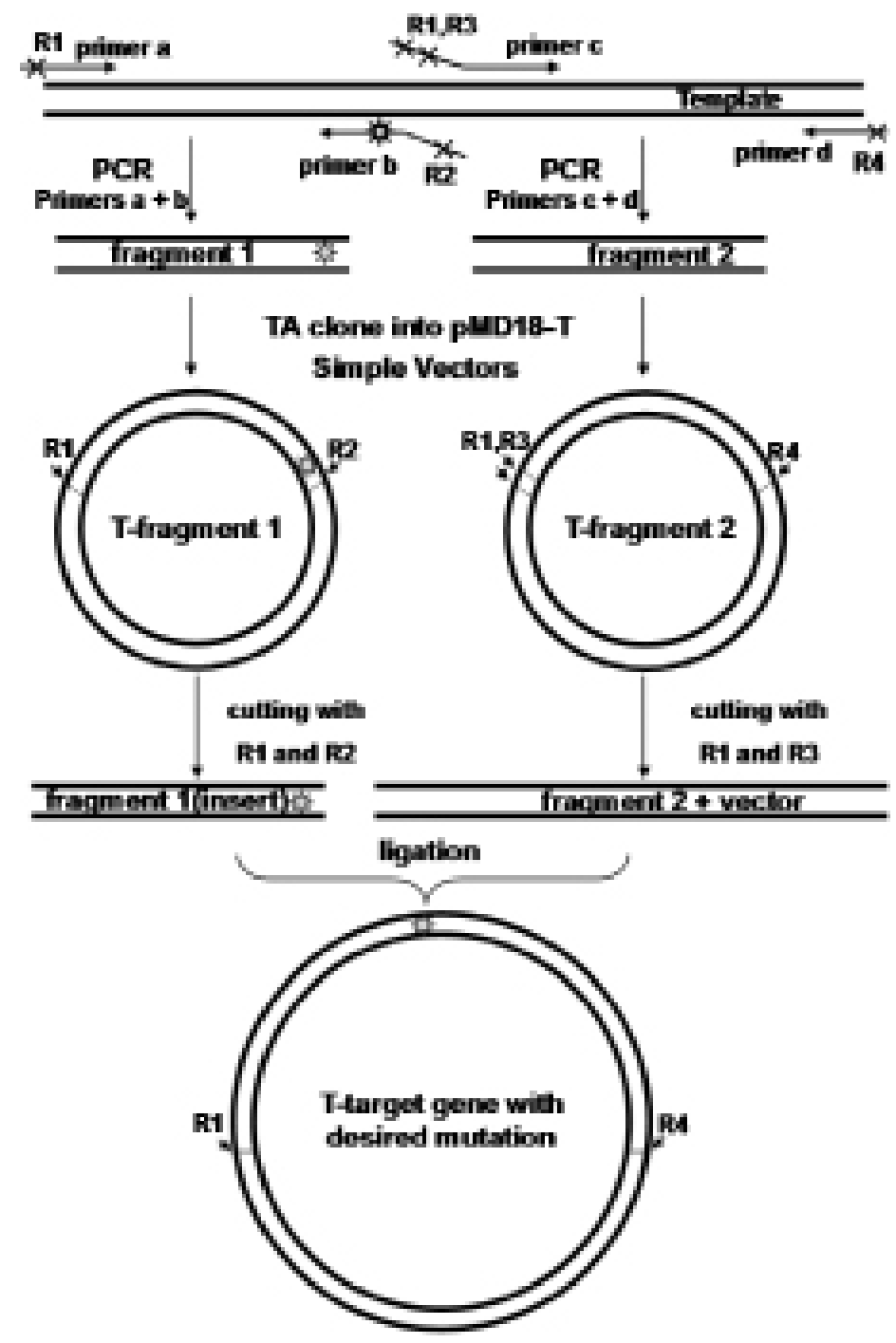

Figure 1. Diagram of site-directed mutagenesis for large gene. Flanking primers (a and d) and mutagenic primers (b and c) were used to generate PCR products (fragments 1 and 2). Then, PCR fragments 1 and 2 were sub-cloned respectively into T-vectors. Finally, full-length gene with desired mutation was created by cutting one fragment (R1 and R2) and inserting it into T-vector (predigested by R1 and R3), which contains another fragment. Stars indicate mutant site. 
To test the applicability of this DNA piecing mutagenesis strategy, we applied the method to generate the $\mathrm{p} 150^{\text {Glued }}$ G59S mutation. We performed PCR in a volume of $50 \mu \mathrm{L}$, which contained $10 \mathrm{ng}$ Flag-p150 Glued plasmid template (Munteanu et al., 2012), 50 pmol of each primer, $20 \mathrm{nmol} \mathrm{dNTP}, 5 \mu \mathrm{L} \mathrm{Mg}^{2+}$ plus buffer, and $5 \mathrm{U}$ LA Taq polymerase (TaKaRa, Shiga, Japan). PCR conditions were as follows (Sang et al., 1996): an initial denaturation step at $94^{\circ} \mathrm{C}$ for $3 \mathrm{~min}$, followed by 5 cycles at $94^{\circ} \mathrm{C}$ for $30 \mathrm{~s}, 48^{\circ} \mathrm{C}$ for $30 \mathrm{~s}$, and $72^{\circ} \mathrm{C}$ for $30 \mathrm{~s}$ (short fragment) or $4 \mathrm{~min}$ (long fragment); then, 25 cycles of $94^{\circ} \mathrm{C}$ for $30 \mathrm{~s}, 55^{\circ} \mathrm{C}$ for $30 \mathrm{~s}$, and $72^{\circ} \mathrm{C}$ for $30 \mathrm{~s}$ (short fragment) or $4 \mathrm{~min}$ (long fragment), and finally 1 cycle of $72^{\circ} \mathrm{C}$ for $10 \mathrm{~min}$. The PCR products of 195 and 3672 bp (Figure 2A) were gel-purified, TA-cloned into pMD18-T Simple Vectors (TaKaRa), transformed into DH5a Escherichia coli cells, and bacterial colonies were screened by digestion with EcoRI and XhoI (Figure 2B). Next, a short fragment was cut from the T-Vector using EcoRI and XhoI as an insert, and sub-cloned into a T-Vector containing the long fragment, which was predigested with EcoRI and SalI. Colonies were screened using EcoRI plus XhoI, and SalI plus XhoI (Figure 2C). Sequencing results demonstrated that the plasmid included the G59S mutation without any other translated amino acid sequence alterations (Figure 2D). Furthermore, the p150 Glued G59S mutant was FLAG-tagged and transfected into HeLa cells. Immunoblotting using an anti-FLAG antibody validated the aggregative expression of $\mathrm{p} 150^{\text {Glued }}$ G59S mutant in transfected HeLa cells (Figure 2E).

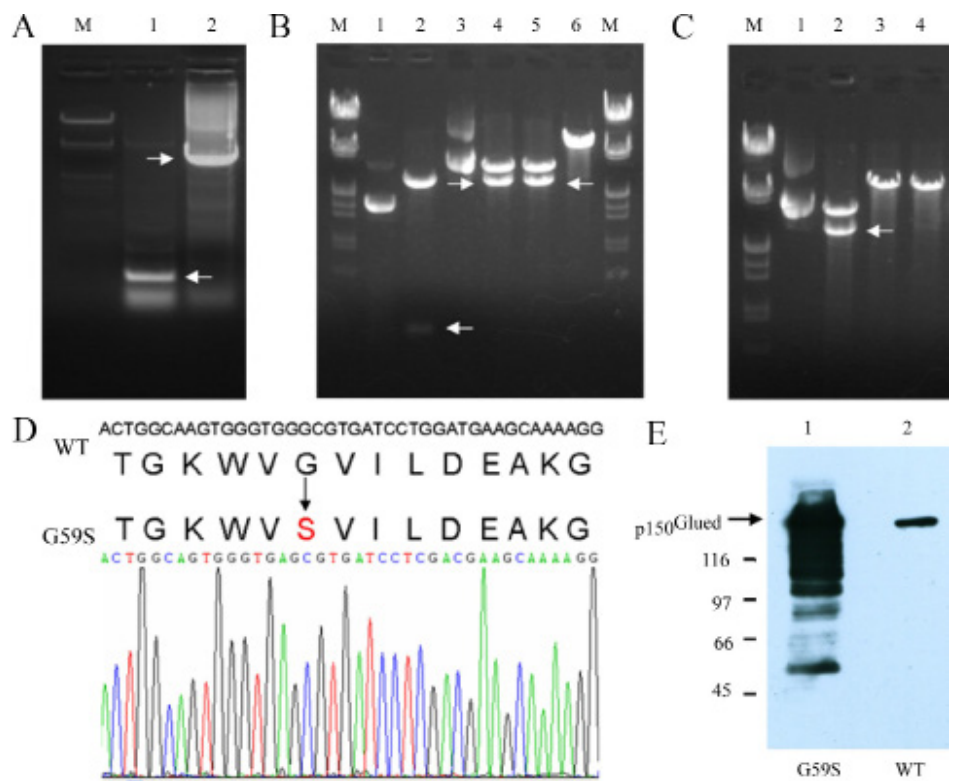

Figure 2. Creating $\mathrm{p} 150^{\mathrm{Glued}} \mathrm{G} 59 \mathrm{~S}$ mutant using DNA cut-paste-based cloning strategy. A. The full-length $\mathrm{p} 150^{\mathrm{Glued}}$ gene was segmented into a small DNA fragment (SDF; lane 1) and a large DNA fragment (LDF; lane 2) by PCR. B. Two DNA fragments were subcloned respectively into pMD18-T Simple Vectors. Miniprep plasmid of T-SDF (lane 1) was verified by restriction enzyme EcoRI/XhoI (lane 2). Miniprep plasmid of T-LDF (lane 3) was verified respectively by restriction enzyme EcoRI/XhoI (lane 4), SalI/XhoI (lane 5) and EcoRI/SalI (lane 6). C. The smaller DNA fragment (cut from T-SDF with EcoRI/SalI) was integrated into T-LDF (digested with EcoRI/SalI) and formed a full-length $\mathrm{p} 150^{\text {Glued }}$ gene with G59S mutation (T-p150 Glued G59S). T-p150 Glued G59S (lane 1) was verified by restriction enzyme EcoRI/XhoI (lane 2), SalI/XhoI (lane 3) and EcoRI/SalI (lane 4) respectively. D. Sequencing result of the $\mathrm{p} 150^{\text {Glued }}$ G59S mutation. E. Anti-FLAG antibody Western blot showing expression of Flag-p150 G59S (lane 1) and Flag-p150 Glued (lane 2) in HeLa cells. 


\section{DISCUSSION}

More than $13 \%$ of gene-coding sequences are larger than 2100 bp (or 700 amino acids) in many species, including human and Drosophila (data not shown). Although few strategies have been developed for large gene site-directed mutagenesis, large genes increase the difficulty of this technique. In this study, we developed a site-directed mutagenesis method for large genes using a DNA CPCS method. Using this approach, site-directed mutagenesis was performed reliably and was validated. Furthermore, we successfully generated a $150^{\text {Glued }}$ G59S mutant. The limitation of our CPCS method is that it is both time- and energy-consuming; however, it provides an alternative method for large gene site-directed mutagenesis as well as gene splicing and deletion.

In conclusion, we developed a novel approach for manipulating large gene site-directed mutagenesis using a CPCS method. Using this method, we successfully constructed a p150 Glued G59S mutant. Moreover, this method can be applied for generating large cDNAs of fusion proteins, chimeric proteins, and insertion or deletion mutagenesis.

\section{ACKNOWLEDGMENTS}

We are grateful to Dr. Xuebiao Yao for critically reading the manuscript and for helpful discussion. Research supported by grants from the Chinese Academy of Science (\#KSCX2YW-R140) and the National Natural Science Foundation of China (\#81300418). Research also supported by grants from the China Postdoctoral Science Foundation (\#2012M511591, \#2013T60708) and The Youth Innovation Fund of the First Affiliated Hospital of Zhengzhou University.

\section{REFERENCES}

Bok JW and Keller NP (2012). Fast and easy method for construction of plasmid vectors using modified quick-change mutagenesis. Methods Mol. Biol. 944: 163-174.

Bu Y, Wang H, Li J, Zhang Z, et al. (2013). Long distance multiple-site directed plasmid mutagenesis by one-step PCR using non-overlapped primers. Mol. Biotechnol. 55: 49-53.

Carrigan PE, Ballar P and Tuzmen S (2011). Site-directed mutagenesis. Methods Mol. Biol. 700: 107-124.

Evans PM and Liu C (2005). SiteFind: a software tool for introducing a restriction site as a marker for successful sitedirected mutagenesis. BMC Mol. Biol. 6: 22.

Heckman KL and Pease LR (2007). Gene splicing and mutagenesis by PCR-driven overlap extension. Nat. Protoc. 2: 924-932.

Hemsley A, Arnheim N, Toney MD, Cortopassi G, et al. (1989). A simple method for site-directed mutagenesis using the polymerase chain reaction. Nucleic Acids Res. 17: 6545-6551.

Higuchi R, Krummel B and Saiki RK (1988). A general method of in vitro preparation and specific mutagenesis of DNA fragments: study of protein and DNA interactions. Nucleic Acids Res. 16: 7351-7367.

Ho SN, Hunt HD, Horton RM, Pullen JK, et al. (1989). Site-directed mutagenesis by overlap extension using the polymerase chain reaction. Gene 77: 51-59.

Hutchison CA 3rd, Phillips S, Edgell MH, Gillam S, et al. (1978). Mutagenesis at a specific position in a DNA sequence. J. Biol. Chem. 253: 6551-6560.

Jin C, Cai X, Ma H, Xue Y, et al. (2007). An efficient site-directed mutagenesis method for ColE1-type ori plasmid. Anal. Biochem. 363: 151-153.

Laird FM, Farah MH, Ackerley S, Hoke A, et al. (2008). Motor neuron disease occurring in a mutant dynactin mouse model is characterized by defects in vesicular trafficking. J. Neurosci. 8: 1997-2005.

Levy JR, Sumner CJ, Caviston JP, Tokito MK, et al. (2006). A motor neuron disease-associated mutation in p150Glued perturbs dynactin function and induces protein aggregation. J. Cell Biol. 172: 733-745. 
Liang X, Peng L, Li K, Peterson T, et al. (2012). A method for multi-site-directed mutagenesis based on homologous recombination. Anal. Biochem. 427: 99-101.

Mitchell LA, Cai Y, Taylor M, Noronha AM, et al. (2013). Multichange isothermal mutagenesis: a new strategy for multiple site-directed mutations in plasmid DNA. ACS Synth Biol. 2: 473-477.

Munteanu B, Braun M and Boonrod K (2012). Improvement of PCR reaction conditions for site-directed mutagenesis of big plasmids. J. Zhejiang Univ. Sci. B 13: 244-247.

Nabavi S and Nazar RN (2005). Simplified one-tube "megaprimer" polymerase chain reaction mutagenesis. Anal. Biochem. 345: 346-348.

Puls I, Jonnakuty C, LaMonte BH, Holzbaur EL, et al. (2003). Mutant dynactin in motor neuron disease. Nat. Genet. 33: 455-456.

Sang N, Condorelli G, De Luca A, MacLachlan TK, et al. (1996). Generation of site-directed mutagenesis by extralong, high-fidelity polymerase chain reaction. Anal. Biochem. 233: 142-144.

Turchin A and Lawler JF Jr (1999). The primer generator: a program that facilitates the selection of oligonucleotides for site-directed mutagenesis. Biotechniques 26: 672-676.

Wei H, Hu J, Wang L, Xu F, et al. (2012). Rapid gene splicing and multi-sited mutagenesis by one-step overlap extension polymerase chain reaction. Anal. Biochem. 429: 76-78.

Yan P, Gao X, Shen W, Zhou P, et al. (2012). Parallel assembly for multiple site-directed mutagenesis of plasmids. Anal. Biochem. 430: 65-67.

Zhang N, Liu J, Ding X, Aikhionbare F, et al. (2007). FBXL5 interacts with p150Glued and regulates its ubiquitination. Biochem. Biophys. Res. Commun. 359: 34-39.

Zoller MJ and Smith M (1982). Oligonucleotide-directed mutagenesis using M13-derived vectors: an efficient and general procedure for the production of point mutations in any fragment of DNA. Nucleic Acids Res. 10: 6487-6500. 